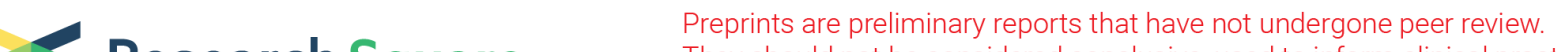 $\begin{array}{ll}\text { Research Square } & \text { They should not be considered conclusive, used to inform clinical practice, } \\ \text { or referenced by the media as validated information. }\end{array}$
}

\section{A case report of late-onset schizophrenia differentiated from a dementing disorder}

\section{Takamitsu Shimada ( $\nabla$ shimadatakamitsu@okabe-net.jp )}

Kanazawa Medical University: Kanazawa Ika Daigaku https://orcid.org/0000-0003-3982-0551

\section{Takashi Uehara}

Department of Neuropsychiatry, Kanazawa Medical University

\section{Tatsuya Nagasawa}

Department of Neuropsychiatry, Kanazawa Medical University

\section{Mitsuru Hasegawa}

Medical Corporation Sekijinkai Okabe Hospital

\section{Yoshiki Maeda}

Medical Corporation Sekijinkai Okabe Hospital,

\section{Yasuhiro Kawasaki}

Department of Neuropsychiatry, Kanazawa Medical University

\section{Case report}

Keywords: cerebrospinal fluid biomarker, dementing disorder, late-onset schizophrenia, neuropathology, risperidone

Posted Date: April 30th, 2021

DOI: https://doi.org/10.21203/rs.3.rs-466697/v1

License: (c) (i) This work is licensed under a Creative Commons Attribution 4.0 International License. Read Full License

Version of Record: A version of this preprint was published at Neurocase on December 23rd, 2021. See the published version at https://doi.org/10.1080/13554794.2021.2016858. 


\section{Abstract \\ Background}

With the increase in life expectancy and the subsequent increase in the older population, the clinical importance of late-onset psychosis also increases. Neuropathological findings play an important role in the differential diagnosis of dementing disorders in older adults. Herein, we report a case of late-onset schizophrenia that required differentiation from a dementing disorder.

\section{Case presentation:}

The patient was an 83-year-old woman who had experienced auditory hallucinations since she was 67 years old. She was hospitalized for treatment of her psychosis. Initially, various examinations were performed to consider the possibility that she had a dementing disorder such as dementia with Lewy bodies. ${ }^{123} \mathrm{I}$-meta-iodobenzylguanidine myocardial scintigraphy revealed no decrease in iodine accumulation in the myocardium, and ${ }^{123}$-ioflupane dopamine transporter imaging revealed no decrease in dopamine transporter accumulation in the striatum. The patient had an elevated concentration of total tau $(488 \mathrm{pg} / \mathrm{mL})$, a cerebrospinal fluid biomarker. After comprehensive testing, the patient was diagnosed with late-onset schizophrenia. Her psychiatric symptoms such as auditory hallucinations diminished after the administration of the recommended first-line drug risperidone ( $3 \mathrm{mg} /$ day), and she was discharged on day 90 .

\section{Conclusions}

This case was identified as late-onset schizophrenia. However, an elevated total tau concentration was observed, indicating that neurofibrillary tangles and neuronal death, which are characteristic of Alzheimer 's disease, may also have been present. Late-onset schizophrenia should be treated based on an appropriate differential diagnosis, including neuropathological consideration of dementing disorders.

\section{Background}

With the increase in life expectancy and the older population, the clinical diagnosis of late-onset psychosis has become important. The pathophysiological background and clinical symptoms of lateonset schizophrenia vary, and it is thought that biological, psychological, social, and environmental factors are involved in its development. The Swiss physician and psychiatrist Manfred Bleuler defined late-onset schizophrenia as schizophrenia that develops over the age of 40 [1]. In the current operational diagnostic criteria from the International Classification of Diseases, Tenth Revision and the Diagnostic and Statistical Manual of Mental Disorders, Fifth Edition, the age of onset is not considered in the diagnosis of schizophrenia; however, young onset should be distinguished from elderly onset in this disease [2]. Although the neuropathological findings of late-onset psychotic disorders have not been fully 
elucidated, there has been remarkable progress in neuropathological methodologies in recent years. Agerelated pathological factors such as neurofibrillary tangles, neuronal death, senile plaques, Lewy bodies, and argyrophilic granules are easily observed, and these neuropathological findings are important for the evaluation of the pathophysiology of late-onset psychotic disorders. Furthermore, schizophrenia itself is a risk factor for the development of dementia [3], and dementia-related diseases need to be considered in the long-term treatment of schizophrenia. We herein report a case of late-onset schizophrenia that differed from a dementing disorder.

\section{Case Presentation}

The patient was an 83-year-old woman. Her highest level of education was junior high school, with an academic performance in the middle of the lower half of students. She exhibited nervous behavior. She had no history of psychiatric disorders, and no one in her family had been diagnosed with such disorders. Her medical history included angina pectoris, adenomatous polyposis, and a left clavicle fracture. However, she had not been diagnosed with any neurological conditions that could have affected her central nervous system, including head trauma with loss of consciousness, active cancer, thyroid disease, epilepsy, seizure, substance-related disorders, current steroid use, or intellectual disability.

At the age of 18, she married her first cousin and subsequently gave birth to two children. Her main job had been to assist her husband with his labor business (construction work, charcoal production, etc.). Her mother-in-law was strict, and therefore, the patient was always nervous. At the age of 67 , after her motherin-law died, the patient began to hear the deceased calling to her at bedtime. From the age of 72, after the death of her husband, the patient lived with their eldest son and his wife. The patient had no problems with daily activities such as meal preparation, grooming, or continence and experienced no forgetfulness. At the age of 82 , the patient less frequently went out and had little social interaction. At the age of 83 , her hallucinations took on the form of blame, i.e., hearing her mother-in-law say, "I died because of you, so I will kill you and take you to hell." At the same time, the patient began to believe that "a large animal was moving under the house." The patient wandered around the house and began constantly hearing her mother-in-law's voice. After 10 days, the patient went to the Department of Neuropsychiatry, Kanazawa Medical University Hospital, and was hospitalized as a psychiatric inpatient for the treatment of her psychosis. The patient's psychiatric symptoms on admission were as follows: 1) auditory hallucinations, persisting for 16 years and suddenly increasing in severity in the previous 10 days; 2) visual hallucinations, including those of animals and bugs; 3 ) persecutory delusions; 4) soliloquies; 5) wandering; and 6) insomnia.

The patient was conscious (Japan Coma Scale: 0, Glasgow Coma Scale: E4V5M5), and her hematological and biochemical data were within normal ranges. Electroencephalography revealed no notable findings, and neurological examinations revealed no notable abnormalities in her cranial nerves. Her deep tendon reflex was neither enhanced nor attenuated, and there was no laterality or resting tremor on either side. Mild muscle rigidity was noted in both upper limbs (right > left). Akinesia, bradykinesia, mask-like face, postural instability, and Myerson's sign were not observed. Table 1 contains the 
neuropsychological test results. The patient's Mini-Mental State Examination score was 26, with deductions for lack of attention and disorientation of time and place. Her total score for Hasegawa's Dementia Scale-Revised was 28 , with deductions for backward digits and memory loss. The patient's Wechsler Adult Intelligence Scale, third edition, results indicated a total intelligence quotient (IQ) of 72 (verbal IQ, 73; performance IQ, 76). She exhibited borderline intellectual functioning, and there was no significant difference between her verbal and performance IQs. Her predicted full-scale IQ on the Japanese Adult Reading Test was 79.8. According to her Wechsler Memory Scale-Revised results, the patient's attention and concentration were particularly low; the other items were at a borderline level. In the noise pareidolia test, her optical-illusion response rate was $5 \%$. A horizontal section obtained by T1weighted magnetic resonance imaging of the patient's head revealed atrophy of the cerebral cortex, dilation of the ventricles, and infarct lesions in the basal ganglia and thalamus (Fig. 1). No hippocampal atrophy was noticeable in the T1-weighted coronal section. Single-photon emission computed tomography of the patient's brain using an easy Z-score imaging system revealed poor blood flow in the temporoparietal junction, precuneus, posterior cingulate gyrus, and occipital lobe (Fig. 2). ${ }^{123}$ l-ioflupane dopamine transporter (DAT) imaging using DATView (Nihon Medi-Physics Co., Ltd., Tokyo, Japan) revealed no loss of dopamine-producing neurons in either striatum; however, there was mild laterality (left > right) (Fig. 3). ${ }^{123}$-meta-iodobenzylguanidine (MIBG) myocardial scintigraphy revealed no decrease in myocardial MIBG accumulation in either early or late images (Fig. 4). General findings from cerebrospinal fluid examination were unremarkable (Table 2). However, the concentration of one cerebrospinal fluid biomarker, total tau (t-tau), measured using an enzyme-linked immunosorbent assay (ELISA) (SRL, Inc., Tokyo, Japan), was elevated. 
Table 1

Neuropsychological test results

\begin{tabular}{|lll|}
\hline Test & Item & Score \\
\hline MMSE & - & 26 \\
\hline HDS-R & - & 28 \\
\hline WAIS-III & Total IQ & 72 \\
\hline & Verbal IQ & 73 \\
\hline Performance IQ & 76 \\
\hline Verbal comprehension index & 80 \\
\hline JART & Perceptual organization index & 91 \\
\hline WMS-R & Working memory index & 79 \\
\hline Pevised; WAIS-III, Wechsler Adult Intelligence Scale, third edition; JART, Japanese Adult Reading Test; & 78 \\
\hline WMS-R, Wechsler Memory Scale-Revised. & 79.8 \\
\hline & Processing speed index & 72 \\
\hline Noise pareidolia test & Verbal memory & 84 \\
\hline & Visual memory & 74 \\
\hline & General memory & 53 \\
\hline & Attention/concentration & 74 \\
\hline & Delayed recall & $38 / 40$ \\
\hline & Correct answer & $2 / 40$ \\
\hline & Pareidolia & $0 / 40$ \\
\hline & Oversight & $5 \%$ \\
\hline & Rate of optical-illusion response & 74 (I) \\
\hline
\end{tabular}


Table 2

Cerebrospinal fluid

\section{General cerebrospinal fluid}

\begin{tabular}{|cl|}
\hline Cell count & $0 / \mu \mathrm{L}$ \\
\hline Polynuclear leukocytes & $0 / \mu \mathrm{L}$ \\
\hline Mononucleosis & $0 / \mu \mathrm{L}$ \\
\hline Other cells & $0 / \mu \mathrm{L}$ \\
\hline Erythrocyte & $56 / \mu \mathrm{L}$ \\
\hline Protein & $44 \mathrm{mg} / \mathrm{dL}$ \\
\hline Glucose & $63 \mathrm{mg} / \mathrm{dL}$ \\
\hline Cl & $125 \mathrm{mEq} / \mathrm{L}$ \\
\hline IgG & $3.6 \mathrm{mg} / \mathrm{dL}$ \\
\hline \%lgG & $8.20 \%$ \\
\hline Albumin & $21 \mathrm{mg} / \mathrm{dL}$ \\
\hline Specific biomarkers & \\
\hline HVA & $58.4 \mathrm{ng} / \mathrm{mL}$ \\
\hline 5-HIAA & $18.5 \mathrm{ng} / \mathrm{mL}$ \\
\hline Total tau & $488 \mathrm{pg} / \mathrm{mL}$ \\
\hline Phosphorylated tau & $45 \mathrm{pg} / \mathrm{mL}$ \\
\hline Amyloid $\beta_{1-40}$ & $2,180 \mathrm{pmol} / \mathrm{L}$ \\
\hline Amyloid $\beta_{1-42}$ & $135 \mathrm{pmol} / \mathrm{L}$ \\
\hline Cl, chlorine; IgG, immunoglobulin G; HVA, homovanillic acid; $5-\mathrm{HIAA}, 5-\mathrm{hydroxyindoleacetic} \mathrm{acid.}$ \\
\hline
\end{tabular}

Figure 5 shows the clinical course of and treatment for the patient. Antipsychotics were administered to treat her psychiatric symptoms, such as auditory and visual hallucinations, persecutory delusion, and insomnia. Quetiapine was started at a low dosage and cautiously increased up to $600 \mathrm{mg} /$ day, in case of adverse events, but the effect was limited. The treatment was changed to aripiprazole, and the dosage increased up to $24 \mathrm{mg} /$ day; however, the drug was ineffective and exacerbated her auditory hallucinations and persecutory delusion, leading to its discontinuation. The treatment was changed to risperidone, with attention to any extrapyramidal symptoms, starting at $2 \mathrm{mg} /$ day; hallucinations almost disappeared after the dosage had been increased to $3 \mathrm{mg} /$ day. To treat the patient's insomnia, quetiapine 
(200 mg/day) was co-administered. Her other psychiatric symptoms were alleviated, and the patient was discharged on day 90 .

\section{Discussion And Conclusions}

In this case, the auditory hallucinations of an 83-year-old woman had persisted from the age of 67, and psychiatric symptoms such as persecutory delusion and insomnia were observed. Although she exhibited no other negative symptoms or social dysfunctions, the patient was diagnosed with late-onset schizophrenia. Compared with early-onset schizophrenia, factors related to late-onset schizophrenia have been reported to include a weaker family history of schizophrenia, better psychosocial functioning, and being female [4], all of which were consistent with this case. In a pathological examination based on an autopsy, Nagao et al. [5] reported that dementia with Lewy bodies (DLB) was observed in $26.1 \%$ and argyrophilic grain dementia (AGD) in $21.7 \%$ of patients who developed schizophrenia and delusional disorder after 40 years of age. Similarly, both DLB and AGD were observed in $36.4 \%$, moderate AD in $18.2 \%$, and mild $A D$ in $9.1 \%$ of patients who developed these symptoms after 65 years of age [5]. In this case, in addition to the patient's advanced age, she had a history of visual hallucinations and mild muscle rigidity was observed; therefore, dementing disorders were considered.

Symptoms of DLB include progressive cognitive impairment that interferes with daily life activities, fluctuation of cognitive functions, visual hallucinations, and parkinsonism [6]. Other features include decreased dopamine transporter uptake in the basal ganglia and decreased iodine uptake in MIBG myocardial scintigraphy [6]. When the rate of optical illusion response is $2.5 \%$ or more, the reported sensitivity and specificity of distinguishing DLB and Alzheimer's disease (AD) are $71 \%$ and $80 \%$, respectively [7]. In this case, findings related to DLB, such as a history of visual hallucinations, muscle rigidity, and a high illusion response rate (5\%), were observed. However, the patient did not exhibit progressive decline in cognitive function that interfered with her daily life, even though it is an essential symptom for a diagnosis of DLB. She also did not exhibit fluctuations in her cognitive function (especially in attention/concentration), another core characteristic of DLB. Furthermore, DAT imaging revealed no decrease in dopamine transporter uptake, and MIBG myocardial scintigraphy indicated no decrease in iodine uptake. From these results, DLB was considered to be unlikely.

AGD is a disease based on the pathological definition reported by Braak et al. in 1987 [8]. Generally, AGD has the following clinical features: 1 ) onset in old age; 2 ) initial symptoms of memory loss, after which behavioral and psychological symptoms such as stubbornness, irritability, persecutory delusion, character change, and violent behavior may be observed; 3) slow progression; 4) limited effect of cholinesterase inhibitors; 5 ) morphologic imaging showing medial lateral atrophy of the anterior temporal lobe, centered on the ambient gyrus, with laterality; 6 ) functional imaging showing a decrease in the medial aspect of the temporal lobe with laterality; and 7) normal concentrations of amyloid $\beta_{1-42}$, $t$-tau, and phosphorylated tau [9-11]. In this case, late onset, slow progression of the disease, and persecutory delusion were consistent with the characteristics of AGD. However, the patient did not exhibit stubbornness, irritability, character change, or violent behavior. In addition, brain morphology and 
functional imaging data were inconsistent, and an elevated t-tau concentration suggested that AGD was unlikely.

As there was no progressive decline in cognitive function in this case, the possibility of $A D$ was low; however, the cerebrospinal fluid findings required further attention. Decreased amyloid $\beta$ and elevated $t-$ tau concentrations are used to diagnose or predict the prognosis of AD [12]. However, the measurement of these markers varies depending on the diagnostic technology and the facility [13]. Based on the results of t-tau measurement using ELISA in 1,031 patients (consisting of 366 with AD, 168 with non-AD dementia, 316 with non-dementia neurological diseases, and 181 healthy controls), the sensitivity and specificity for AD were $59.1 \%$ and $89.5 \%$, respectively, with a cutoff concentration of t-tau set at $>375$ $\mathrm{pg} / \mathrm{mL}$ [14]. In our case, the t-tau concentration was $488 \mathrm{pg} / \mathrm{mL}$, higher than the aforementioned cutoff value. Elevated tau protein levels are thought to reflect changes in neurofibrils and neuronal cell death [12]. It has been reported that cerebrospinal fluid biomarkers matched the characteristics of $A D$ in $36 \%$ of older participants without cognitive impairment [15]. Therefore, even in the absence of apparent cognitive decline, it is possible that cerebrospinal fluid biomarkers may be present at abnormal concentrations if histological changes are under way. The patient in this case was not clinically diagnosed with AD, although the neuropathological findings suggested that neurofibrillary tangles and neuronal death, which are characteristic of $A D$, might have been present.

Regarding pharmacotherapy for late-onset schizophrenia, the use of atypical antipsychotics is recommended, although the recommendation is not based on the highest levels of evidence such as from randomized controlled trials or meta-analyses. Risperidone (1.25-3.5 mg/day) is recommended as the first-line drug for late-onset schizophrenia, and quetiapine (100-300 mg/day), olanzapine (7.5$15 \mathrm{mg} /$ day), and aripiprazole (15-30 mg/day) are recommended as second-line drugs [16]. These recommendations are based on questionnaire-based surveys of American experts on their treatment of older adults with mental illnesses, not on clinical evidence. It should be noted that, in our case, quetiapine and aripiprazole did not clearly reduce the patient's hallucinations/delusions, and risperidone, the recommended first-line drug, was effective in treating these symptoms.

This case was identified as late-onset schizophrenia based on clinical symptoms, imaging results, and neuropsychological findings, and treatment with risperidone was effective. However, neuropathologically, it is possible that lesions indicative of a dementing disorder were present. Late-onset schizophrenia should be treated based on an appropriate differential diagnosis, with consideration of dementing disorders as possible comorbidities.

\section{Declarations}

\section{Ethics approval and consent to participate}

Not applicable.

\section{Consent for publication}


Written informed consent was obtained from the patient for the publication of this case report.

\section{Availability of data and materials}

The dataset(s) supporting the conclusions of this article is(are) included within the report.

\section{Competing interests}

The authors declare that they have no competing interests.

\section{Funding}

Not applicable.

\section{Authors' contributions}

TS performed initial and follow-up psychiatric care, contributed to the interpretation of the data, wrote the first draft of the manuscript, and contributed to the editing of the final manuscript. TU and YK contributed to the conception and design. TN, MH, and YM were involved in the literature review and corrections. All authors read and approved the final manuscript.

\section{Acknowledgements}

We would like to thank the patient and her family who participated in this study.

\section{Abbreviations}

IQ, intelligence quotient; AGD, argyrophilic grain disease; AD, Alzheimer's disease; DLB, dementia with Lewy bodies; DAT, dopamine transporter; MIBG, meta-iodobenzylguanidine.

\section{References}

1. Bleuler M. The clinical picture in late schizophrenia. Fortschr Neurol Psychiatr. 1943;15:259-90

2. Howard R, Rabins PV, Seeman MV, Jeste DV. Late-onset schizophrenia and very-late-onset schizophrenia-like psychosis: an international consensus. The International Late-Onset Schizophrenia Group. Am J Psychiatry. 2000;157:172-8

3. Cai L, Huang J. Schizophrenia and risk of dementia: a meta-analysis study. Neuropsychiatr Dis Treat. 2018;14:2047-55

4. Chen L, Selvendra A, Stewart A, Castle D. Risk factors in early and late onset schizophrenia. Compr Psychiatry. 2018;80:155-62

5. Nagao S, Yokota O, Ikeda C, Takeda N, Ishizu H, Kuroda S, et al. Argyrophilic grain disease as a neurodegenerative substrate in late-onset schizophrenia and delusional disorders. Eur Arch Psychiatry Clin Neurosci. 2014;264:317-31 
6. McKeith IG, Boeve BF, Dickson DW, Halliday G, Taylor JP, Weintraub D, et al. Diagnosis and management of dementia with Lewy bodies: fourth consensus report of the DLB Consortium. Neurology. 2017;89:88-100

7. Yokoi K, Nishio Y, Uchiyama M, Shimomura T, lizuka O, Mori E. Hallucinators find meaning in noises: pareidolic illusions in dementia with Lewy bodies. Neuropsychologia. 2014;56:245-54

8. Braak H, Braak E. Argyrophilic grains: characteristic pathology of cerebral cortex in cases of adult onset dementia without Alzheimer changes. Neurosci Lett. 1987;76:124-7

9. Japanese Society of Neurology. Dementia Disease Practice Guidelines. Chapter 11. Retrieved from: https://www.neurology-jp.org/guidelinem/degl/degl_2017_11.pdf. (in Japanese). [accessed 23 Mar 2021]. In: Argyrophilic grain dementia. CQ11-2; 2017

10. Togo T, Isojima D, Akatsu H, Suzuki K, Uchikado H, Katsuse $\mathrm{O}$, et al. Clinical features of argyrophilic grain disease: a retrospective survey of cases with neuropsychiatric symptoms. Am J Geriatr Psychiatry. 2005;13:1083-91

11. Adachi T, Saito Y, Hatsuta H, Funabe S, Tokumaru AM, Ishii K, et al. Neuropathological asymmetry in argyrophilic grain disease. J Neuropathol Exp Neurol. 2010;69:737-44

12. Japanese Society of Neurology. Dementia Disease Practice Guidelines. Chapter 6 . Retrieved from: https://www.neurology-jp.org/guidelinem/degl/degl_2017_06.pdf. (in Japanese). [accessed 23 Mar 2021]. In: Alzheimer's dementia. CQ6-5; 2017

13. Mattsson N, Andreasson U, Persson S, Carrillo MC, Collins S, Chalbot S, et al; Alzheimer's Association QC Program Work Group. CSF biomarker variability in the Alzheimer's Association quality control program. Alzheimers Dement. 2013;9:251-61

14. Shoji M, Matsubara E, Murakami T, Manabe Y, Abe K, Kanai M, et al. Cerebrospinal fluid tau in dementia disorders: a large scale multicenter study by a Japanese study group. Neurobiol Aging. 2002;23:363-70

15. De Meyer G, Shapiro F, Vanderstichele H, Vanmechelen E, Engelborghs S, De Deyn PP, et al. Diagnosis-independent Alzheimer disease biomarker signature in cognitively normal elderly people. Arch Neurol. 2010;67:949-56

16. Alexopoulos GS, Streim J, Carpenter D, Docherty JP, Expert Consensus Panel for Using Antipsychotic Drugs in Older Patients. Using antipsychotic agents in older patients. J Clin Psychiatry. 2004;65;Suppl 2:5-99; discussion 100-2; quiz 103-4

\section{Figures}


a

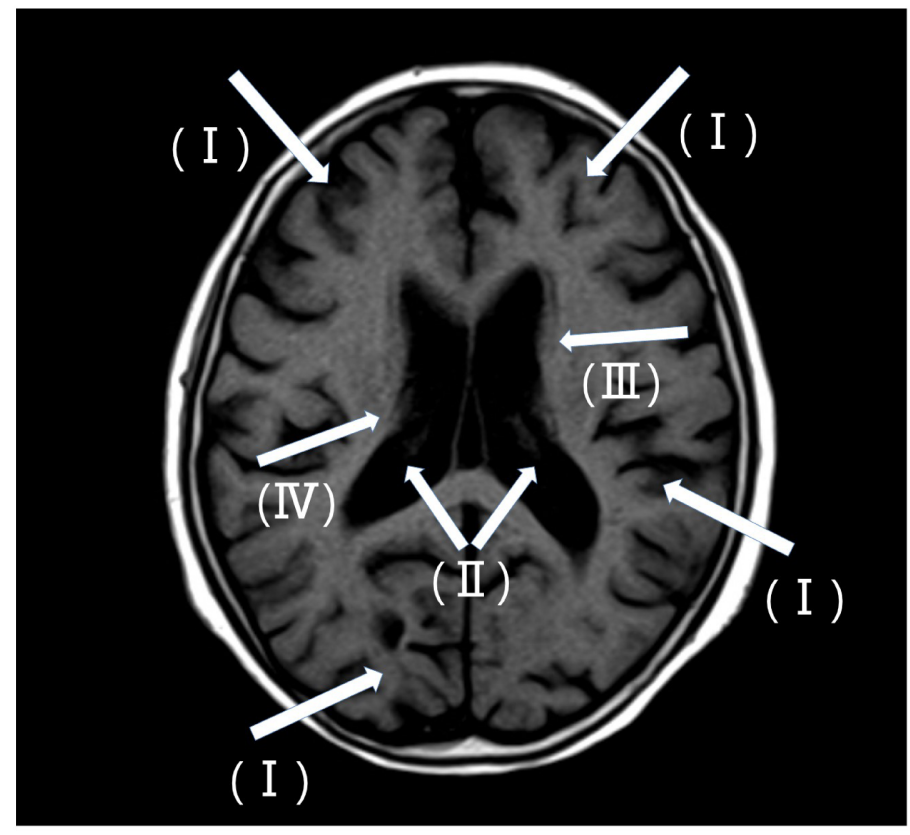

$\mathrm{R}$ b

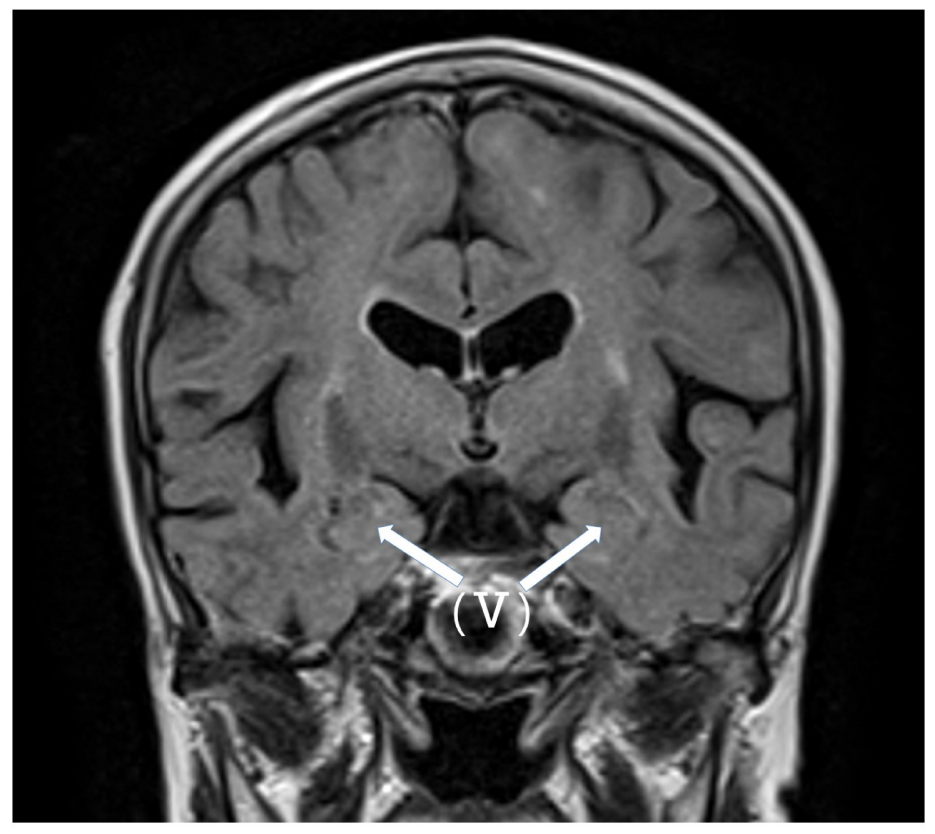

$\mathrm{R}$

Figure 1

Brain structure imaged by T1-weighted magnetic resonance images of the patient's head. a. The horizontal section reveals atrophy of the cerebral cortex (I), dilation of the ventricles (II), and infarct lesions in the basal ganglia (III) and thalamus (IV). b. The coronal section reveals no hippocampal (V) atrophy.
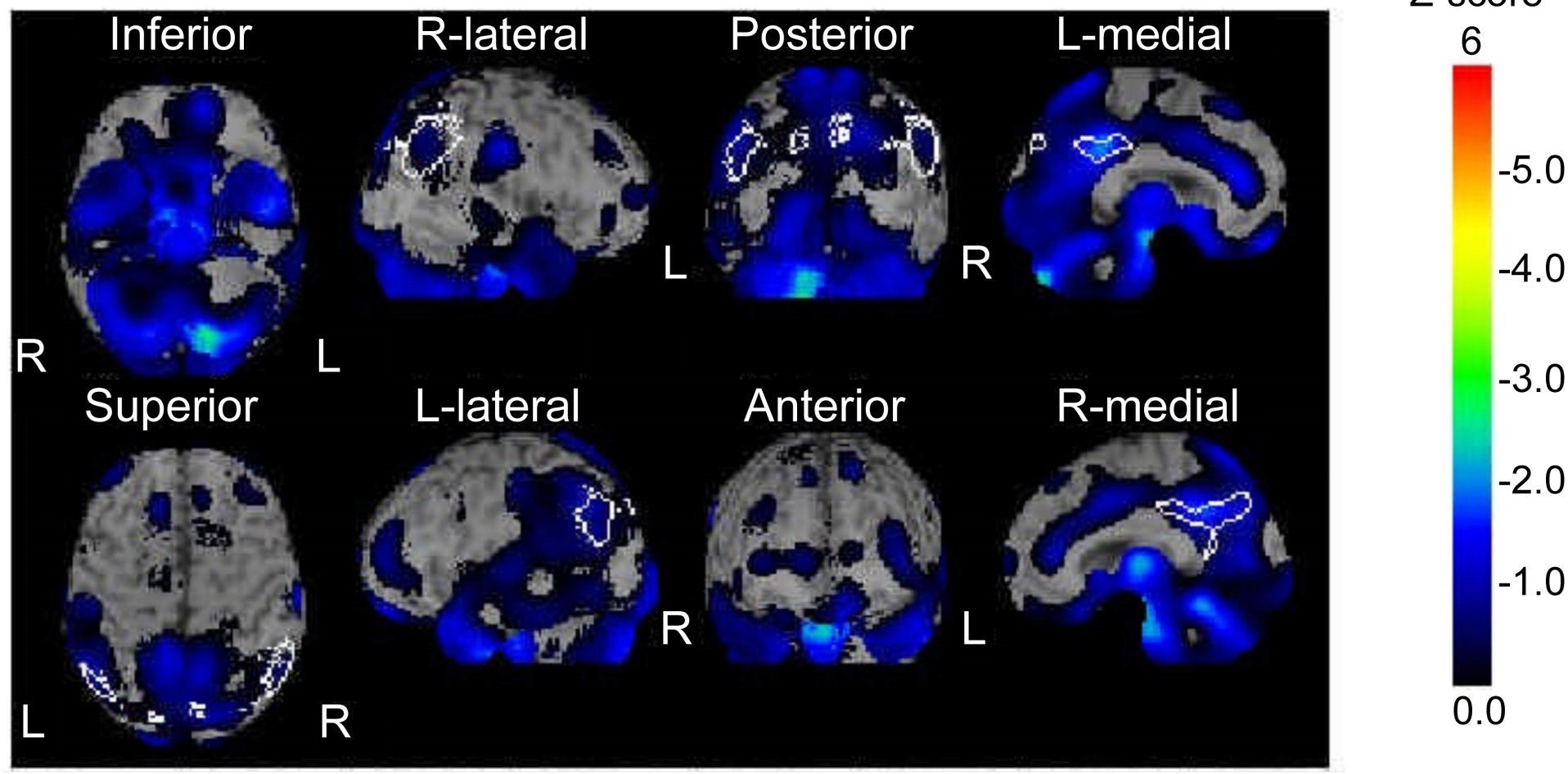

Figure 2 
Single-photon emission computed tomography of the patient's brain using an easy Z-score imaging system The blood flow in the temporoparietal junction, precuneus, posterior cingulate gyrus, and occipital lobe is poor. The regions of poor blood flow that are characteristic of Alzheimer's disease (the anterior cingulate cortex, precuneus, and parietal lobe) are indicated using dashed lines.

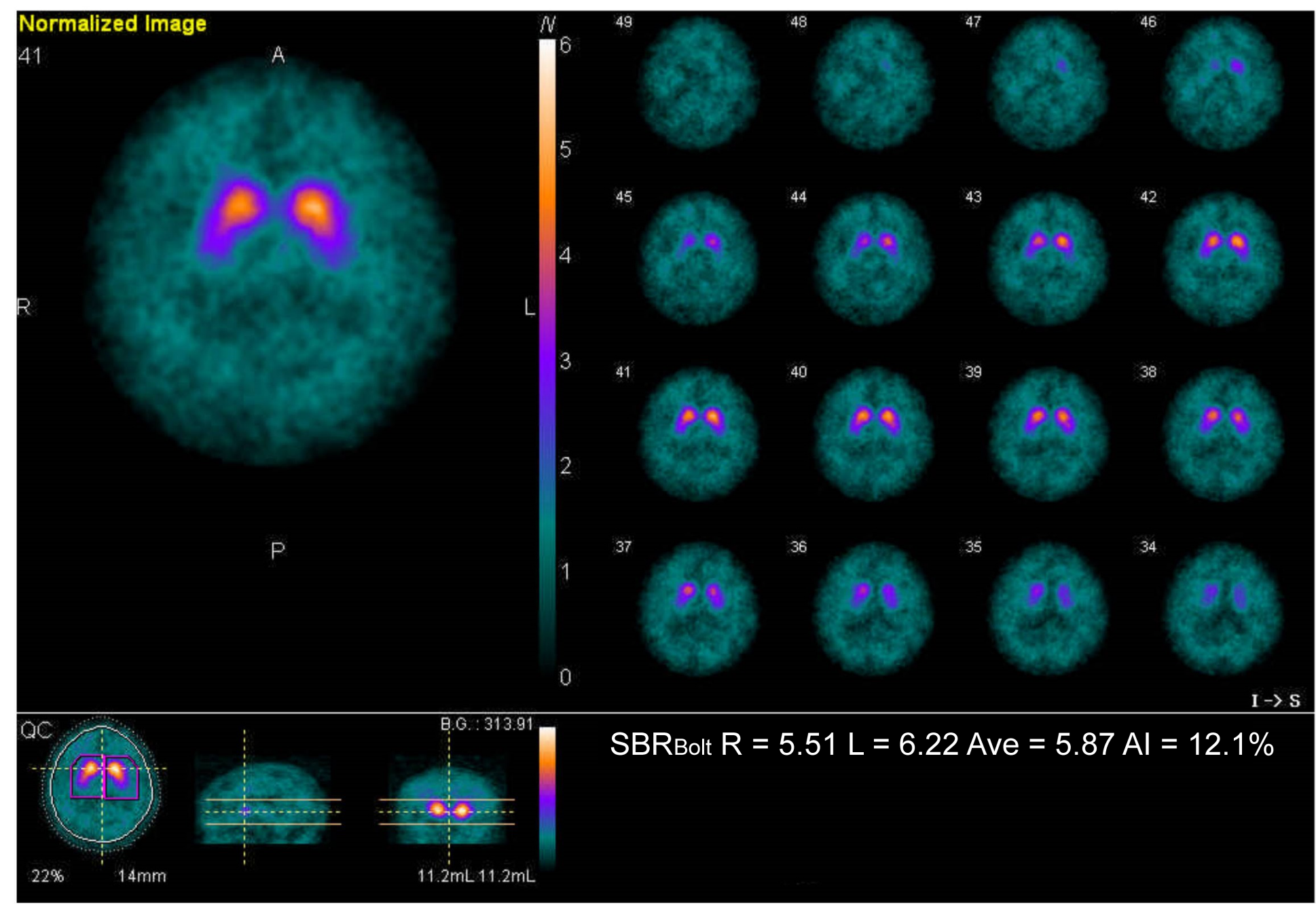

\section{Figure 3}

123I-ioflupane dopamine transporter imaging The images show an increase in 123I-ioflupane accumulation, with a comma-shaped pattern, and mild laterality (left > right). The specific binding ratio in the striatum was 5.51 on the right, 6.22 on the left, and 5.87 on average. The asymmetry index was $12.1 \%$. 


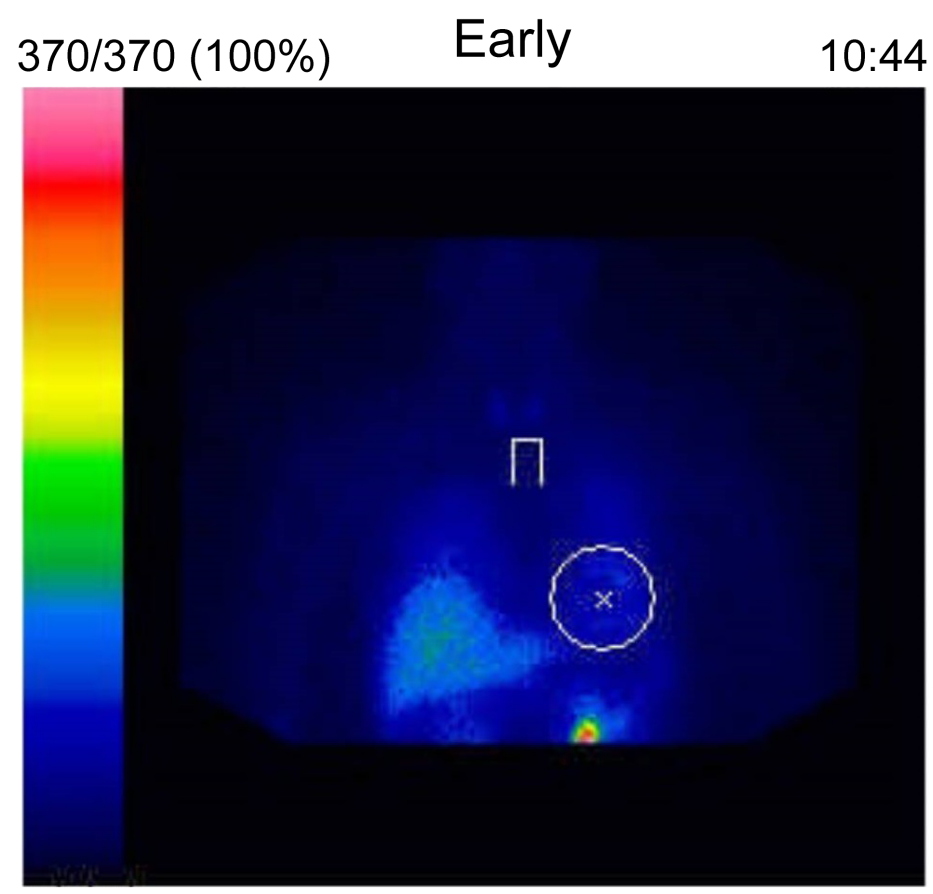

$0 / 0(0 \%)$
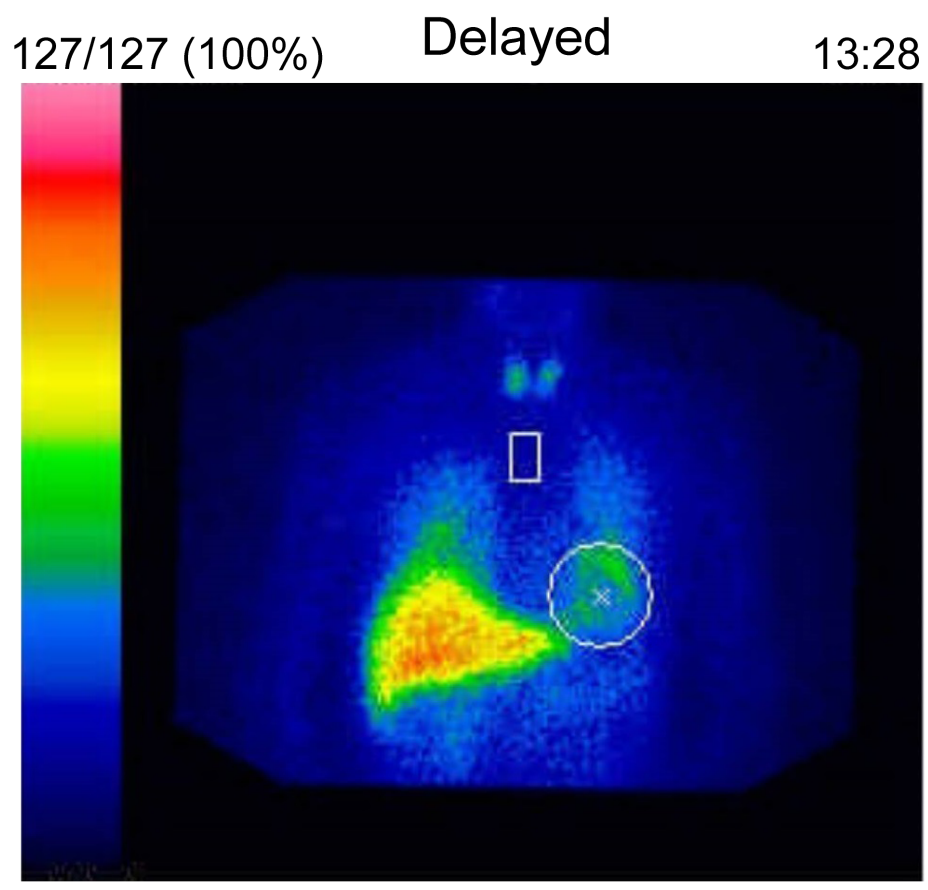

$0 / 0(0 \%)$

\section{Figure 4}

123I-meta-iodobenzylguanidine (MIBG) myocardial scintigraphy No decrease in myocardial MIBG accumulation was observed in either early or late images. Early image: heart, 67.2 counts/pixel; mediastinum, 34.5 counts/pixel; heart-to-mediastinum (H/M) ratio, 2.74 (standardized medium energy (ME), standardized reference value $\geq 2.2$ ); Delayed image: heart, 43.7 counts/pixel; mediastinum, 22.5 counts/pixel; H/M ratio, 2.74 (standardized ME, standardized reference value $\geq 2.2$ ). 
Psychiatric symptoms

Hallucination

Persecutory delusion

Disquiet/hyperkinesia

Insomnia

\section{Pharmacotherapy}

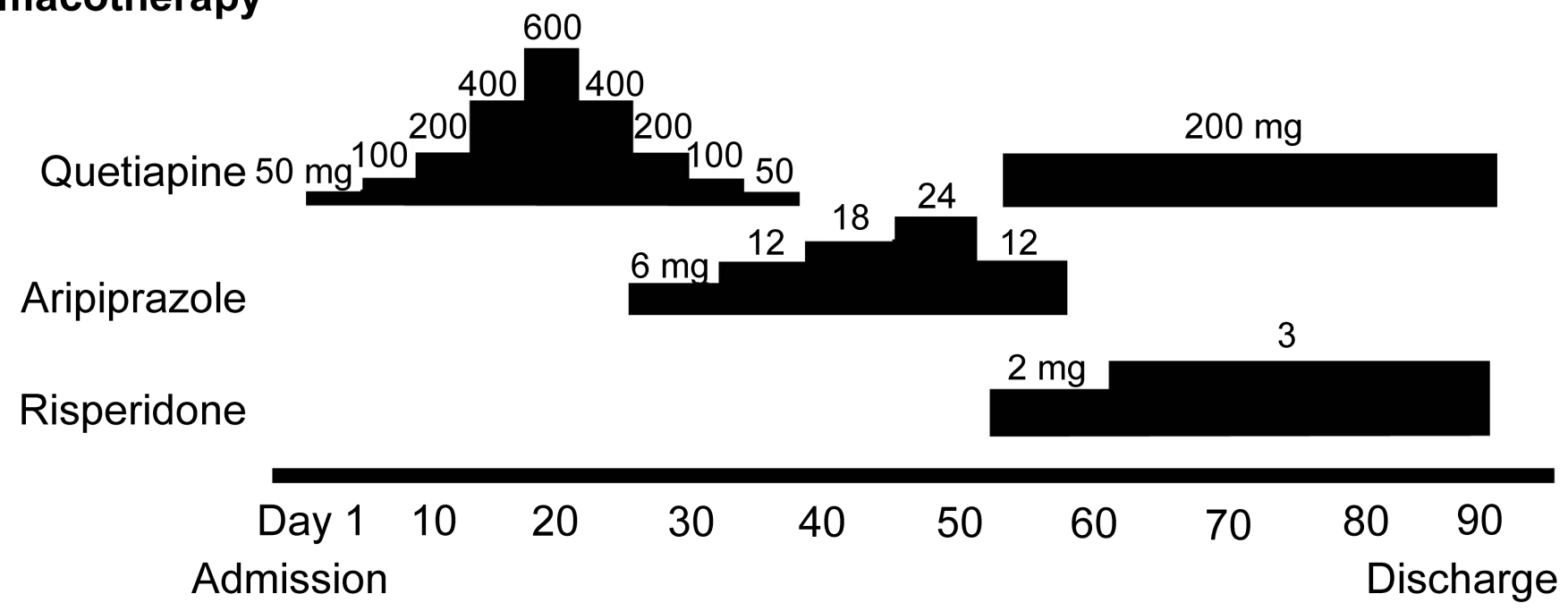

Figure 5

Clinical course and treatment of the patient's psychiatric symptoms 